\title{
Comparing Intelligence Test Profiles to Assess Tourette's Disorder with Attention-Deficit Hyperactivity Disorder
}

\author{
Woo Hyun Kim ${ }^{1}$, Tae Won Park ${ }^{1,2}$, Juhyun Park ${ }^{3}$, Sang-Keun Chung ${ }^{1,2}$, Jong-Chul Yang ${ }^{1,2}$, \\ Jong-II Park ${ }^{4}$, Eun-Ji Kim ${ }^{1,2}$, Eun-Cheong $\mathrm{Cho}^{4}$, and Jae Cheol Park ${ }^{5}$ \\ ${ }^{1}$ Department of Psychiatry, Chonbuk National University Medical School, Jeonju, Korea \\ ${ }^{2}$ Research Institute of Clinical Medicine of Chonbuk National University-Biomedical Research Institute of Chonbuk National University \\ Hospital, Jeonju, Korea \\ ${ }^{3}$ Seoul National University College of Medicine, Seoul, Korea \\ ${ }^{4}$ Department of Psychiatry, Chonbuk National University Hospital, Jeonju, Korea \\ ${ }^{5}$ Department of Statistics, Chonbuk National University, Jeonju, Korea
}

Objectives: The study compared the intelligence test profiles of Tourette's Disorder (TD), attention-deficit hyperactivity disorder (ADHD), and TD with ADHD (TD+ADHD) groups.

Methods: The Korean Wechsler Intelligence Scale for Children-third edition (K-WISC-III) and Korean Wechsler Intelligence Scale for Children-fourth edition (K-WISC-IV) were administered to 13 children and adolescents with TD, 17 children and adolescents with ADHD, and 15 children and adolescents with TD+ADHD. Each parameter was compared among the groups using the Kruskal-Wallis test. Results: The mean scores of the freedom from distractibility/working memory index (FD/WMI) and the digit span and arithmetic subtests of the TD+ADHD group were significantly lower than those of the TD group.

Conclusion: According to the intelligence test results, the comorbid ADHD+TD group showed a significant decrease in working memory compared to the TD group. These findings are similar to those of previous research on cognitive functions and suggest that the TD+ADHD comorbid and TD alone groups exhibit different endophenotypes. The results also imply that WISC-III and WISC-IV, the most commonly used intelligence tests clinically, are effective in evaluating cognitive functions such as attention. Further research is required to confirm these results.

Key Words: Tourette's disorder; Attention-deficit hyperactivity disorder; Intelligence test; Working memory function.

Received: June 22, 2016 / Revision: September 5, 2016 / Accepted: September 26, 2016

Address for correspondence: Tae Won Park, Department of Psychiatry, Chonbuk National University Medical School, 20 Geonji-ro, Deokjin-gu, Jeonju 54907, Korea

Tel: +82-63-250-2028, Fax: +82-63-275-3157, E-mail: ptaewon@jbnu.ac.kr

\section{INTRODUCTION}

Tourette's disorder (TD) is a severe form of tic disorder, characterized by multiple motor and vocal tics that persist for at least 1 year. The pathogenesis of TD is unclear; however, it is presumed to be associated with the interaction of genetic and environmental factors during the development of the central nervous system. Abnormalities in the cortico-striato-thalamic circuit are one of the most widely cited factors associated with TD.1) Neuropsychological impairment observed in TD directly affects learning abilities and manifests as symptoms related to tic disorder and other comorbidities. ${ }^{2)}$ To clarify the mechanism behind such neuropsychological disabilities, ongoing research has focused on the neuropsychological char-

This is an Open Access article distributed under the terms of the Creative Commons Attribution Non-Commercial License (http://creativecommons.org/licenses/by-nc/3.0) which permits unrestricted non-commercial use, distribution, and reproduction in any medium, provided the original work is properly cited. acteristics of TD. TD is known to negatively affect sustained attention, ${ }^{3)}$ visuomotor integration, ${ }^{4)}$ reaction time and verbal fluency, ${ }^{5)}$ problem-solving, ${ }^{6}$ and response inhibition. ${ }^{7}$

Attention-deficit hyperactivity disorder (ADHD), characterized by attention deficiency, impulsivity, and hyperactivity, is known to be the most common psychiatric disorder in childhood. Previous studies have shown that children with ADHD have impaired planning and inhibiting abilities as well as damaged verbal working memory. ${ }^{8,9)}$ Such reduced inhibiting abilities are known to extend to executive functions, such as working memory, cognitive flexibility, planning and organizing, and verbal fluency. ${ }^{10,11)}$

Clinically, ADHD and tic disorder are often comorbid. ADHD symptoms occur in about $50 \%$ of patients with tic disorder, while tic disorder symptoms occur in about 10$20 \%$ of patients with ADHD. ${ }^{12)}$ In a study on cognitive abilities and emotional or behavioral problems in children with TD 
with or without the coexistence of ADHD, it was found that children with comorbid TD and ADHD have reduced cognitive abilities and more severe emotional or behavioral problems. However, the exact mechanisms behind such associations remain unknown.

The results of previous neuropsychological studies that compared the comorbid TD and ADHD comorbid group to TD alone group or $\mathrm{ADHD}$ alone group are not consistent. According to Carter et al., ${ }^{13)}$ the children in the comorbid TD and ADHD group face greater difficulties with regard to their external and internal symptoms as well as social adjustment compared to those in the TD alone group. Moreover, Greimel et al. ${ }^{14)}$ have found that, although the ADHD group and ADHD+TD group have poorer executive functions compared to the general population, ironically, the ADHD+TD group performed better than the $\mathrm{ADHD}$ group. This led to the conclusion that $\mathrm{ADHD}$ alone acts as the negative factor, whereas the presence of TD does not affect the concentration ability of the patients. Sukhodolsky et al. ${ }^{15)}$ found that while the ADHD group exhibited considerable neuropsychological impairment, this was not the case with the TD alone and normal control groups. Due to the inconsistent results, which depended on the researcher and the type of comorbid disease, a firm conclusion on the association between TD and ADHD is yet to be established.

Thus far, the Wechsler Intelligence Scale for Children (WISC) has often been used to evaluate the cognitive abilities of children. WISC-III comprises 13 subtests, with the introduction of a new subtest called symbol research in addition to the 12 subtests that were previously used in the Wechsler Intelligence Scale for Children-Revised [WISC-R; Korean Educational Developmental Institute (KEDI)-WISC is the Korean version of WISC-R]. ${ }^{16)}$ The 13 subtests assess full intelligence quotient (IQ), verbal IQ, and functional IQ. ${ }^{17)}$ Until recently, WISC-III has been used clinically, while WISC-IV (2003) was recently standardized for Korean children. Therefore, both Korean Wechsler Intelligence Scale for Children-third edition (K-WISC-III) and Korean Wechsler Intelligence Scale for Children-fourth edition (K-WISC-IV) are in use. ${ }^{18)}$ WISCIV is characterized by its emphasis on the measurement of working memory and information processing speed. Subtests and indicators for processing speed, working memory, and fluid reasoning, which are considered to be the most important cognitive abilities in many clinical research studies, have been added or improved. ${ }^{18)}$

In a previous study using WISC-III, the comorbid TD and ADHD group showed that working memory index (WMI) and arithmetic subtest scores were significantly lower, but there was not much difference in scores between the TD group and the control group. However, the ADHD group was not included in the study, thereby presenting limitations. ${ }^{19)}$ In another study that administered WISC-III in ADHD patients, the attention factor and processing speed factor indices, which constitute two of the four factor-based indices, were found to be significantly lower compared to normal controls but higher than that of the TD group. ${ }^{20)}$

Few research studies have used WISC-III and WISC-IV to compare the comorbid group, TD alone group, and ADHD group together. In this study, K-WISC-III and K-WISC-IV were used to compare and evaluate the differences between the comorbid group and alone groups and determine the cognitive characteristics of the comorbid group.

\section{METHODS}

\section{Participants}

Participants were recruited from the Department of Psychiatry, Chonbuk National University Hospital. We initially selected children aged 6-14 years, who met the criteria set in DSM-IV-TR for TD, ADHD, or comorbid comorbid TD and ADHD. Patients with initial visit records that contained the results of the ADHD rating scale, ${ }^{21)} \mathrm{K}-\mathrm{WISC}-\mathrm{III}$, or K-WISCIV were primarily selected as subjects for the study, which comprised 45 patients. The exclusion criteria were as follows: a history of pervasive developmental disorder, learning disorder, mood disorders, anxiety disorder, and psychotic disorders; a history of organic brain disease, seizures, or other neurological disorders; and an IQ below 70. Patients taking any psychiatric medication including stimulants, which can affect the cognitive function, were also excluded.

\section{Research tools}

\section{K-WISC-III}

This test was standardized to be adapted for Korean children by Kwak et al. ${ }^{17)}$ based on WISC-III. The test is suitable for children aged 6 to 16 years and comprises verbal subtests and performance tests, which provide not only the complete IQ score but also verbal IQ and performance IQ scores. A subject's cognitive abilities can be inferred from the converted score profiles of each subtest. The verbal test assessed a child's ability to assimilate information; detect similarities; perform arithmetic, vocabulary, and comprehension tasks; and digit span (i.e., supplementary subtests). The performance test comprised tasks based on picture completion, picture arrangement, block design, object assembly, coding and symbol search, and mazes (i.e., supplementary subtests).

\section{K-WISC-IV}

WISC-IV, which is the newly revised fourth edition of the 
intelligence test for children developed by Wechsler, was standardized by Kwak et al. ${ }^{18)}$ and adapted for the assessment of Korean children. WISC-IV lacks three subtests that have previously been included in WISC-III. Instead, WISC-IV has additional five new subtests (picture concepts, letter-number sequencing, matrix reasoning, cancellation, word reasoning), as well as full scale IQ scores and four indicators (verbal comprehension, perceptual reasoning, working memory, processing speed). In particular, subtests and indicators to measure indicators such as the processing speed, working memory, and fluid reasoning, which are some of the most important cognitive abilities highlighted in several clinical research studies, have been added or improved.

Test comparing the KEDI-WISC results of the comorbid group is present, but WISC-III or WISC-IV results is not much. In this paper, we apply WISC-IV with a lot of similarities in the
WISC-III test items.

In this study, subtests known to have the same scores in both K-WISC-III and K-WISC-IV and their summed score indices were directly compared. ${ }^{22)}$

\section{Analysis methods}

In this study, Sexual differences were analyzed by Fisher exact test, Full Scale Intelligence Quotient (FSIQ), the four index scores, and the scores of each subtest of both K-WISC-III and K-WISC-IV administered to the TD, ADHD, and comorbid TD and ADHD groups were collected. These scores were obtained in order to observe the differences in the cognitive abilities of the groups in each specific domain. Furthermore, the Kruskal-Wallis test was used to compare the groups, while the Mann-Whitney test method was used for post-hoc analysis. The only statistical program used was SPSS 16.0 (SPSS

Table 1. Demographic and clinical variables of subjects by group

\begin{tabular}{|c|c|c|c|c|}
\hline Variables & Comorbid TD and ADHD $(n=15)$ & $\operatorname{TD}(n=13)$ & $\operatorname{ADHD}(n=17)$ & Chi-square \\
\hline Age (yrs) & & & & $6.951(p=0.031)$ \\
\hline $8.93(2.27)$ & $8.27(2.37)$ & $10.31(1.89)$ & $8.47(2.18)$ & \\
\hline ARS & & & & $1.804(p=0.179)$ \\
\hline $23.67(11.4)$ & $21.60(9.90)$ & & $26.88(11.52)$ & \\
\hline Gender & & & & $1.742(p=0.533)$ \\
\hline Male & $13(86.7 \%)$ & $13(100 \%)$ & $15(88.2 \%)$ & \\
\hline Female & $2(13.3 \%)$ & $0(0 \%)$ & $2(11.8 \%)$ & \\
\hline
\end{tabular}

ADHD: attention-deficit hyperactivity disorder, ARS: ADHD Rating Scale, SD: standard deviation, TD: Tourette's disorder

Table 2. Scores of K-WISC-III and K-WISC-IV by group

\begin{tabular}{|c|c|c|c|c|c|c|}
\hline \multirow[t]{2}{*}{ Scores } & $\begin{array}{l}\text { Comorbid TD and } \\
\text { ADHD }(n=15)\end{array}$ & $\operatorname{TD}(n=13)$ & $\operatorname{ADHD}(n=17)$ & \multirow{2}{*}{$\begin{array}{l}\text { Chi- } \\
\text { square }\end{array}$} & \multirow[t]{2}{*}{$P$} & \multirow[t]{2}{*}{ Post hoc } \\
\hline & Mean (SD) & Mean (SD) & Mean (SD) & & & \\
\hline $\mathrm{FIQ/FSIQ}$ & $87.73(12.37)$ & $99.92(18.81)$ & $89.00(11.23)$ & 3.578 & 0.167 & \\
\hline $\mathrm{VC} / \mathrm{VCl}$ & $89.93(14.27)$ & $98.62(18.55)$ & $92.06(14.84)$ & 1.506 & 0.471 & \\
\hline Similarities & $7.93(2.58)$ & $9.46(3.50)$ & $8.71(2.80)$ & 2.030 & 0.362 & \\
\hline Vocabulary & $9.33(2.61)$ & $10.15(3.85)$ & $10.35(3.18)$ & 0.727 & 0.695 & \\
\hline Information & $8.73(3.67)$ & $11.77(3.86)$ & $8.94(3.60)$ & 5.488 & 0.064 & \\
\hline Comprehension & $7.40(2.53)$ & $8.77(3.03)$ & $7.06(2.89)$ & 2.591 & 0.274 & \\
\hline $\mathrm{PO} / \mathrm{PRI}$ & $93.27(9.48)$ & $102.31(15.12)$ & $92.53(14.95)$ & 2.317 & 0.314 & \\
\hline Block design & $9.73(1.67)$ & $10.62(3.82)$ & $9.94(3.58)$ & 0.448 & 0.799 & \\
\hline Picture completion & $7.47(2.30)$ & $9.00(2.89)$ & $8.12(3.22)$ & 1.947 & 0.378 & \\
\hline FD/WMI & $86.53(14.13)$ & $104.00(16.63)$ & $91.00(17.26)$ & 8.397 & 0.015 & Comorbid TD and ADHD $<$ TD \\
\hline Digit span & $7.73(3.33)$ & $11.31(3.12)$ & $8.41(3.84)$ & 7.251 & 0.027 & Comorbid TD and ADHD $<$ TD \\
\hline Arithmetic & $7.40(3.70)$ & $10.69(3.07)$ & $9.65(2.15)$ & 7.426 & 0.024 & Comorbid TD and ADHD $<$ TD \\
\hline PSI & $89.87(12.11)$ & $95.23(11.59)$ & $89.82(14.16)$ & 1.942 & 0.379 & \\
\hline Coding & $8.87(2.56)$ & $9.31(2.25)$ & $7.71(2.85)$ & 3.081 & 0.214 & \\
\hline Symbol search & $7.47(3.80)$ & $9.15(2.61)$ & $8.94(2.41)$ & 3.150 & 0.207 & \\
\hline
\end{tabular}

ADHD: attention-deficit hyperactivity disorder, FD: freedom from distractibility, FIQ: Full Scale IQ, FSIQ: Full Scale Intelligence Quotient, K-WISC-III: Korean Wechsler Intelligence Scale for Children-third edition, K-WISC-IV: Korean Wechsler Intelligence Scale for Children-fourth edition, PO: Perceptual Organization, Post-hoc: Mann-Whitney test, PRI: perceptual reasoning index, PS: processing speed, PSI: processing speed index, SD: standard deviation, TD: Tourette's disorder, VC: verbal comprehension, VCl: verbal comprehension index, WMl: working memory index 
Inc., Chicago, IL, USA) for Windows, with the statistical significance set at $\mathrm{p}<0.05$.

\section{RESULTS}

\section{Demographic characteristics}

There were 45 subjects in total, with 15,13 , and 17 subjects in the comorbid TD and ADHD, TD, and ADHD groups, respectively. Their characteristics are shown in Table 1. While the gender difference in the three groups was not significant $\left(\chi^{2}=1.742, p=0.533\right)$, the mean age of the TD group was significantly higher than the other groups $(\mathrm{p}=0.031)$. There was no significant difference in the $\mathrm{ADHD}$ rating scale between the the comorbid TD and ADHD and ADHD alone group $(\mathrm{p}=0.179)$.

\section{K-WISC comparison between comorbid TD and $\mathrm{ADHD}, \mathrm{TD}$, and ADHD groups}

After performing the Kruskal-Wallis test on the K-WISC results, we found significant differences between the three groups in the freedom from distractibility (FD)/WMI score $(\mathrm{p}=0.015)$ and in the two subtests, digit span $(\mathrm{p}=0.027)$ and arithmetic $(\mathrm{p}=0.024)$ (Table 2). The remaining indices and subtests did not show any significant differences. In the case of Full Scale IQ/FSIQ, there was no significant difference. However, in this case, the scores of K-WISC-III and K-WISCIV were compared equally. Therefore, further studies regarding the direct comparison between the two tests are needed. To observe the differences between the three groups in greater detail, we conducted the Mann-Whitney test for post-hoc analysis. As a result, the comorbid TD and ADHD group showed significantly lower scores in the FD/WMI index, digit span subtest, and arithmetic subtest compared to the TD group ( $\mathrm{p}=0.002, \mathrm{p}=0.005, \mathrm{p}=0.018$, respectively).

In all the measured indices, there were no significant differences between the comorbid TD and ADHD and ADHD groups and between the TD and ADHD groups.

\section{DISCUSSION}

In this study, the K-WISC scores of TD, ADHD, and comorbid TD and ADHD comorbid groups were compared. After comparing the three groups, it was found that the TD+ADHD comorbid group showed significantly lower scores than TD alone group in two subtests of the FD/WMI index: digit span and arithmetic. This result is similar to the results of Debes et al..$^{19)}$ In their study, the score of the arithmetic subtest of WMI was significantly lower in the comorbid TD and ADHD group than in the TD alone group. Moreover, the processing speed index (PSI) results were also similar: the TD alone group and comorbid TD and ADHD comorbid group did not show significant differences in our study. However, their results of the digit span subtest differ from ours in that there was no significant difference between the TD and comorbid groups.

In one study that compared the TD and $\mathrm{ADHD}$ groups to normal controls using WISC-III, the ADHD group scored significantly lower in the digit span subtest compared to normal controls. However, the TD group did not. ${ }^{23)}$ This result suggests that the psychopathology of ADHD might be the cause of the dysfunction shown by the comorbid TD and ADHD group. However, this remains to be seen as the comorbid TD and ADHD group was not included in the study. Meanwhile, Shin et al. ${ }^{24)}$ used some neuropsychological test and the Korean version of the WISC-R to compare TD, ADHD, comorbid TD and ADHD, and normal control groups. In their study, scores of cognitive indices including intelligence test were similarly low in both the comorbid TD and ADHD and $\mathrm{ADHD}$ groups, whereas the scores of the TD alone group did not differ much from the normal control group. This implies that $\mathrm{ADHD}$, rather than $\mathrm{TD}$, plays a negative role in the comorbid group. Mahone et al. ${ }^{25)}$ and Roessner et al. ${ }^{26)}$ found that ADHD and comorbid TD and ADHD groups, but not the TD group, exhibited attention deficiency. However, they also found that the comorbid TD and ADHD group did not perform better than the ADHD group and claimed that, in comorbid patients, ADHD is the key factor that affects neuropsychological execution, independent of TD symptoms. Moreover, the high comorbidity rate of ADHD in the TD group ${ }^{12)}$ may imply that TD can possibly express diverse phenotypes. ${ }^{27)}$ Furthermore, as the volume decrease of amygdala in TD is an associated symptom of ADHD, further clinical and imaging studies are required. ${ }^{28)}$

In addition, although not significant, scores differed in the overall index in the order of comorbid TD and ADHD $<\mathrm{ADHD}<\mathrm{TD}$. This pattern should be further studied due to two reasons. First, previous studies on ADHD groups have found that ADHD reduces the working memory and processing speed indices of WISC. ${ }^{29)}$ Second, earlier studies on comorbid TD and ADHD groups suggested that ADHD, but not TD, was the major negative factor. ${ }^{15,25,26)}$ These two findings imply that ADHD is the dominant negative factor for reduced working memory and processing speed indices in comorbid TD and ADHD comorbid groups. This also implies that there would be minute differences in the working memory and PSI scores between comorbid TD and ADHD and ADHD groups, as TD would barely affect the two indices. However, the scores of working memory in our study was in the order of comorbid $\mathrm{TD}$ and $\mathrm{ADHD}<\mathrm{ADHD}<\mathrm{TD}$. As previous studies have found that TD is the major negative factor of procedural memory, whereas ADHD further affects motor skills and executive functions related to work- 
ing memory, ${ }^{30)}$ future studies that determine why the comorbid TD and ADHD group has a lower WMI score compared to the ADHD group are required.

We have found significant differences between TD, ADHD, and comorbid TD and ADHD groups in subtests of WMI using K-WISC-III and K-WISC-VI, which are the most widely used intelligence tests clinically; this implies that they are very helpful in diagnosis. In particular, our study excluded patients who took psychiatric medication (e.g., psychostimulants), since such drugs might interfere with the test results.

Our study's limitations are as follows:

- First, a normal control group was not included in the study.

- Second, this study was conducted retrospectively with medical records and psychological test reports of the patients.

- Third, as the onset age of ADHD is relatively earlier compared to TD, some of the members of the ADHD group in this study might show signs of TD later. In other words, a true comorbid TD and ADHD patient may have been incorrectly classified as an ADHD patient while conducting the study.

- Lastly, we assumed that the K-WISC-III and K-WISCIV tests are identical. Previous studies have found the index scores of the two tests to be largely similar, but this finding remains controversial and caution is needed in interpreting the results.

\section{CONCLUSION}

In this study, we have confirmed that FD/WMI is significantly lowered in children with comorbid TD and ADHD comorbidity. These findings are similar to that of previous research on cognitive functions and suggest that the comorbid TD and ADHD comorbid and TD alone groups exhibit different endophenotypes. The results also imply that WISCIII and WISC-IV, the most commonly used intelligence tests clinically, are effective in evaluating cognitive functions such as attention. Further research is required in this regard.

\section{Conflicts of Interest}

The authors have no financial conflicts of interest.

\section{REFERENCES}

1) Singer HS. Tourette's syndrome: from behaviour to biology. Lancet Neurol 2005;4:149-159.

2) Comings DE, Himes JA, Comings BG. An epidemiologic study of Tourette's syndrome in a single school district. J Clin Psychiatry 1990;51:463-469.

3) Sherman EM, Shepard L, Joschko M, Freeman RD. Sustained attention and impulsivity in children with Tourette syndrome: comorbidity and confounds. J Clin Exp Neuropsychol 1998;20:644-657.
4) Schultz RT, Carter AS, Gladstone M, Scahill L, Leckman JF, Peterson BS, et al. Visual-motor integration functioning in children with Tourette syndrome. Neuropsychology 1998;12:134-145.

5) Schuerholz LJ, Baumgardner TL, Singer HS, Reiss AL, Denckla MB. Neuropsychological status of children with Tourette's syndrome with and without attention deficit hyperactivity disorder. Neurology 1996;46:958-965.

6) Channon S, Crawford S, Vakili K, Robertson MM. Real-life-type problem solving in Tourette syndrome. Cogn Behav Neurol 2003; 16:3-15.

7) Müller SV, Johannes S, Wieringa B, Weber A, Müller-Vahl K, Matzke $\mathrm{M}$, et al. Disturbed monitoring and response inhibition in patients with Gilles de la Tourette syndrome and co-morbid obsessive compulsive disorder. Behav Neurol 2003;14:29-37.

8) Pennington BF, Ozonoff S. Executive functions and developmental psychopathology. J Child Psychol Psychiatry 1996;37:51-87.

9) Shallice T, Marzocchi GM, Coser S, Del Savio M, Meuter RF, Rumiati RI. Executive function profile of children with attention deficit hyperactivity disorder. Dev Neuropsychol 2002;21:43-71.

10) Willcutt EG, Doyle AE, Nigg JT, Faraone SV, Pennington BF. Validity of the executive function theory of attention-deficit/hyperactivity disorder: a meta-analytic review. Biol Psychiatry 2005;57:13361346.

11) Anderson P. Assessment and development of executive function (EF) during childhood. Child Neuropsychol 2002;8:71-82.

12) Spencer T, Biederman J, Harding M, O'Donnell D, Wilens T, Faraone S, et al. Disentangling the overlap between Tourette's disorder and ADHD. J Child Psychol Psychiatry 1998;39:1037-1044.

13) Carter AS, O'Donnell DA, Schultz RT, Scahill L, Leckman JF, Pauls DL. Social and emotional adjustment in children affected with Gilles de la Tourette's syndrome: associations with ADHD and family functioning. Attention deficit hyperactivity disorder. J Child Psychol Psychiatry 2000;41:215-223.

14) Greimel E, Wanderer S, Rothenberger A, Herpertz-Dahlmann B, Konrad K, Roessner V. Attentional performance in children and adolescents with tic disorder and co-occurring attention-deficit/hyperactivity disorder: new insights from a $2 \times 2$ factorial design study. J Abnorm Child Psychol 2011;39:819-828.

15) Sukhodolsky DG, Landeros-Weisenberger A, Scahill L, Leckman JF, Schultz RT. Neuropsychological functioning in children with Tourette syndrome with and without attention-deficit/hyperactivity disorder. J Am Acad Child Adolesc Psychiatry 2010;49:1155-1164.

16) Park KS, Yoon JY, Park HJ, Park HJ, Kwon KW. Korean Educational Development Institute-Wechsler Intelligence Scale for Children. Seoul: Korean Educational Development Institute;1991.

17) Kwak KJ, Park HW, Kim CT. Manual for the Korean Wechsler Intelligence Scale for Children-III. Seoul: Special Education;2001.

18) Kwak KJ, Oh SW, Kim CT. Korean-Wechsler Intelligence Scale for Children. 4th ed. Seoul: Hakjisa;2011. p.11-13.

19) Debes NM, Lange T, Jessen TL, Hjalgrim H, Skov L. Performance on Wechsler Intelligence Scales in children with Tourette syndrome. Eur J Paediatr Neurol 2011;15:146-154.

20) Snow JB, Sapp GL. WISC-III subtest patterns of ADHD and normal samples. Psychol Rep 2000;87:759-765.

21) So YK, Noh JS, Kim YS, Ko SG, Koh YJ. The reliability and validity of Korean parent and teacher ADHD Rating Scale. J Korean Neuropsychiatr Assoc 2002;41:283-289.

22) Sattler JM. Assessment of children: cognitive foundations. 5th ed. San Diego, CA: Jerome M Sattler;2008.

23) Kang H, Lee SI, Lee AR, Kim SG, Jung HY. Functional impairment and executive dysfunction of children with Tourette disorder: comparison with attention-deficit hyperactivity disorder and non-affected controls. J Korean Acad Child Adolesc Psychiatry 2015;26:190196.

24) Shin MS, Chung SJ, Hong KEM. Comparative study of the behav- 
ioral and neuropsychologic characteristics of tic disorder with or without attention-deficit hyperactivity disorder (ADHD). J Child Neurol 2001;16:719-726.

25) Mahone EM, Cirino PT, Cutting LE, Cerrone PM, Hagelthorn KM, Hiemenz JR, et al. Validity of the behavior rating inventory of executive function in children with ADHD and/or Tourette syndrome. Arch Clin Neuropsychol 2002;17:643-662.

26) Roessner V, Becker A, Banaschewski T, Rothenberger A. Executive functions in children with chronic tic disorders with/without ADHD: new insights. Eur Child Adolesc Psychiatry 2007;16 Suppl 1:36-44.

27) Randolph C, Hyde TM, Gold JM, Goldberg TE, Weinberger DR. Tourette's syndrome in monozygotic twins. Relationship of tic severity to neuropsychological function. Arch Neurol 1993;50:725-
728.

28) Ludolph AG, Pinkhardt EH, Tebartz van Elst L, Libal G, Ludolph AC, Fegert JM, et al. Are amygdalar volume alterations in children with Tourette syndrome due to ADHD comorbidity? Dev Med Child Neurol 2008;50:524-529.

29) Koh M, Noh EA, Kim HW. Korean Wechsler Intelligence Scale for children-fourth edition profiles in child and adolescent with attention-deficit hyperactivity disorder: retrospective study. J Korean Acad Child Adolesc Psychiatry 2015;26:183-189.

30) Rothenberger A, Roessner V, Banaschewski T, Leckman JF. Co-existence of tic disorders and attention-deficit/hyperactivity disorder-recent advances in understanding and treatment. Eur Child Adolesc Psychiatry 2007;16 Suppl 1:1-4. 